\title{
Right-to-left inverted single lung transplantation
}

Masayuki Chida, MD, PhD, Osamu Araki, MD, PhD, Yoko Karube, MD, PhD, and

Sumiko Maeda, MD, PhD, Mibu, Japan

From the Department of General Thoracic Surgery, Dokkyo Medical University School of Medicine, Mibu, Japan. Disclosures: The authors reported no conflicts of interest.

The Journal policy requires editors and reviewers to disclose conflicts of interest and to decline handling or reviewing manuscripts for which they may have a conflict of interest. The editors and reviewers of this article have no conflicts of interest.

Received for publication July 27, 2020; revisions received July 27, 2020; accepted for publication Aug 10, 2020; available ahead of print Sept 30, 2020.

Address for reprints: Masayuki Chida, MD, PhD, 880 Kitakobayashi, Mibu, Shimotsuga, Tochigi 321-0293, Japan (E-mail: chidaths@dokkyomed.ac.jp).

JTCVS Techniques 2020;4:395-7

2666-2507

Copyright (C) 2020 The Authors. Published by Elsevier Inc. on behalf of The American Association for Thoracic Surgery. This is an open access article under the CC BY-NC-ND license (http://creativecommons.org/licenses/bync-nd/4.0/).

https://doi.org/10.1016/j.xjtc.2020.08.081

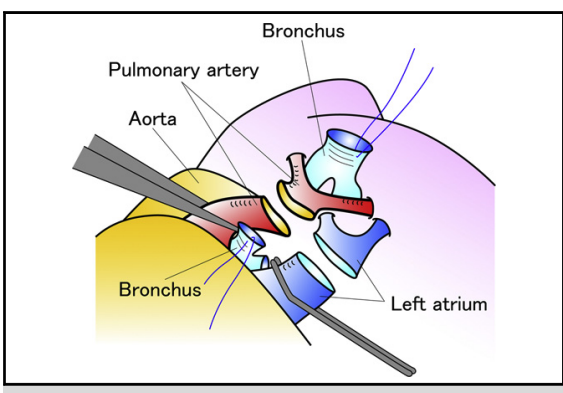

Right-to-left inverted single-lung transplantation procedure via an open-space technique.

\section{CENTRAL MESSAGE \\ Right-to-left inverted single lung \\ transplantation is anatomically \\ feasible. An open space behind \\ the bronchi can be made for \\ pulmonary artery anastomosis \\ by pulling both up.}

See Commentaries on pages 398,399 , and 401
Donor shortage is a critical issue in some areas; thus, singlelung transplantation remains popular. In most countries, a scoring system has been established that allows for the neediest patients to have priority for receiving a donor organ, and it is important to perform lung transplantation using any available lung in those cases. In some patients with uneven disease laterality, transplantation for the worst side is usually suitable, although waiting for a specific side may lose the opportunity for lung transplantation. In contrast, those patients may benefit by undergoing any-side lung donation to increase the opportunity for rapid transplantation, even though the available organ is from an unsuitable side. We report a case of inverted single-lung transplantation in a patient with a lung condition showing uneven laterality (Video 1).

\section{CASE REPORT}

A 56-year-old male patient with idiopathic pulmonary fibrosis was registered in the Japan Organ Transplant Network as a candidate for single-lung transplantation. Lung perfusion scintigraphy showed an uneven accumulation of radioisotopes, right to left $97 \%$ to $3 \%$, whereas blood gas analysis revealed arterial carbon dioxide tension at 63.1 torr and arterial oxygen tension at 162 torr with $5 \mathrm{~L} /$ min of oxygen provided. Furthermore, echocardiogram results indicated secondary pulmonary hypertension with $79 \mathrm{~mm} \mathrm{Hg}$ of estimated systolic pulmonary artery pressure without findings of heart remodeling, indicating that singlelung transplantation would be effective. At 11 months after registration, an opportunity for lung transplantation was presented. Another recipient with priority received the left lung of the donor, whereas the present patient received the right, and we performed a right-to-left inverted lung transplantation procedure. Written informed consent to

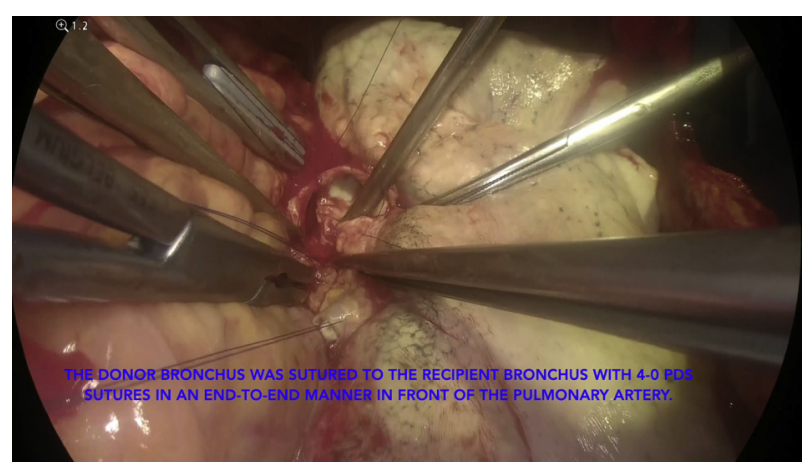

VIDEO 1. Right-to-left inverted single-lung transplantation. Video available at: https://www.jtcvs.org/article/S2666-2507(20)30557-5/fulltext. 

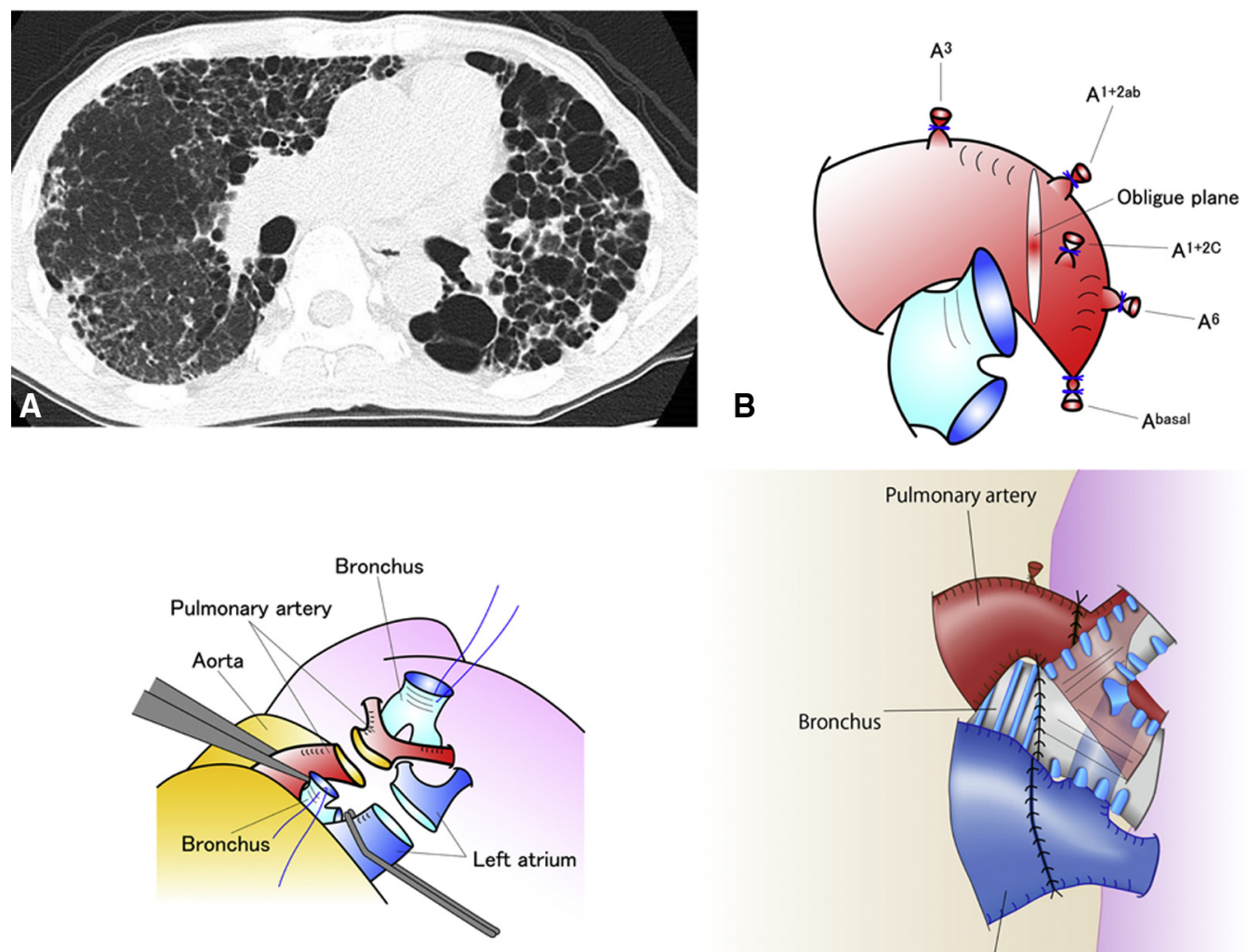

C

D

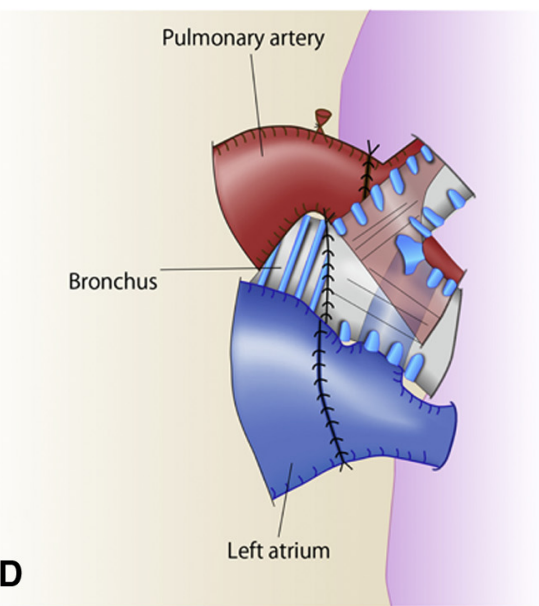

FIGURE 1. A, Preoperative chest computed tomography scan results showed uneven disease laterality. B, Recipient pulmonary artery preparation. The pulmonary artery was divided at the periphery of A6 to leave it beyond the bronchus stump, with ligation of A3, A1+2ab, and A1+2c. The recipient pulmonary artery was cut in an oblique plane, from A1+2ab to A6, so as to obtain a wide site for anastomosis of the right-side main pulmonary artery of the donor. C, Anastomosis of the pulmonary artery was performed after making space by retraction of both the donor and recipient bronchi. D, Final image of hilar anastomosis.

undergo an inverted lung transplantation procedure was obtained from the patient.

\section{Precision Preparation}

The left chest cavity was entered through the fourth intercostal space following an anterior thoracotomy with sternal transection. Venoarterial extracorporeal membrane oxygenation was used due to secondary pulmonary hypertension following confirmation with a pulmonary artery clumping test. Under a left pneumonectomy, the pulmonary artery was divided at the periphery of A6 to leave the pulmonary artery beyond the bronchus stump, with ligation of $\mathrm{A} 3, \mathrm{~A} 1+2 \mathrm{ab}$, and $\mathrm{A} 1+2 \mathrm{c}$ (Figure 1). The right lung graft was then placed in the left thorax of the recipient after rotating the graft from its anatomic position to $180^{\circ}$ around the superior-inferior axis. Thus, the anatomic posterior aspect of the right lung graft became anterior in the left chest of our patient.

\section{Step 1: Pulmonary Artery Anastomosis Behind Bronchi}

Initially, stay sutures were placed on both the recipient and donor bronchi, then pulled to create a space for pulmonary artery anastomosis behind the bronchi. The recipient pulmonary artery was cut in an oblique plane, from $\mathrm{A} 1+2 \mathrm{ab}$ to $\mathrm{A} 6$, so as to obtain a wide anastomotic site for the right-side main pulmonary artery of the donor. The oblique plane of the recipient pulmonary artery was then sutured to that of the donor using 5-0 PROLENE sutures (Ethicon Inc, Tokyo, Japan) beyond the recipient bronchus, which was pulled using a stay suture. The donor bronchus was also retracted using a stay suture to expose the donor pulmonary artery.

\section{Step 2: Bronchial and Left Atrial Anastomosis}

The bronchi stay sutures were removed and the donor bronchus was sutured to the recipient bronchus with 40 PDS sutures (Ethicon Inc) in an end-to-end manner in front of the pulmonary artery. For this type of procedure, it is recommended that both donor and recipient bronchi be cut along an oblique plane, ${ }^{1}$ because the axis angles differ. The membrane part of each bronchus was located on the opposite side; thus, each part was sutured to the cartilage portion of the bronchus. Finally, we pulled out the donor left atrium on the dorsal side of the hilum, then 


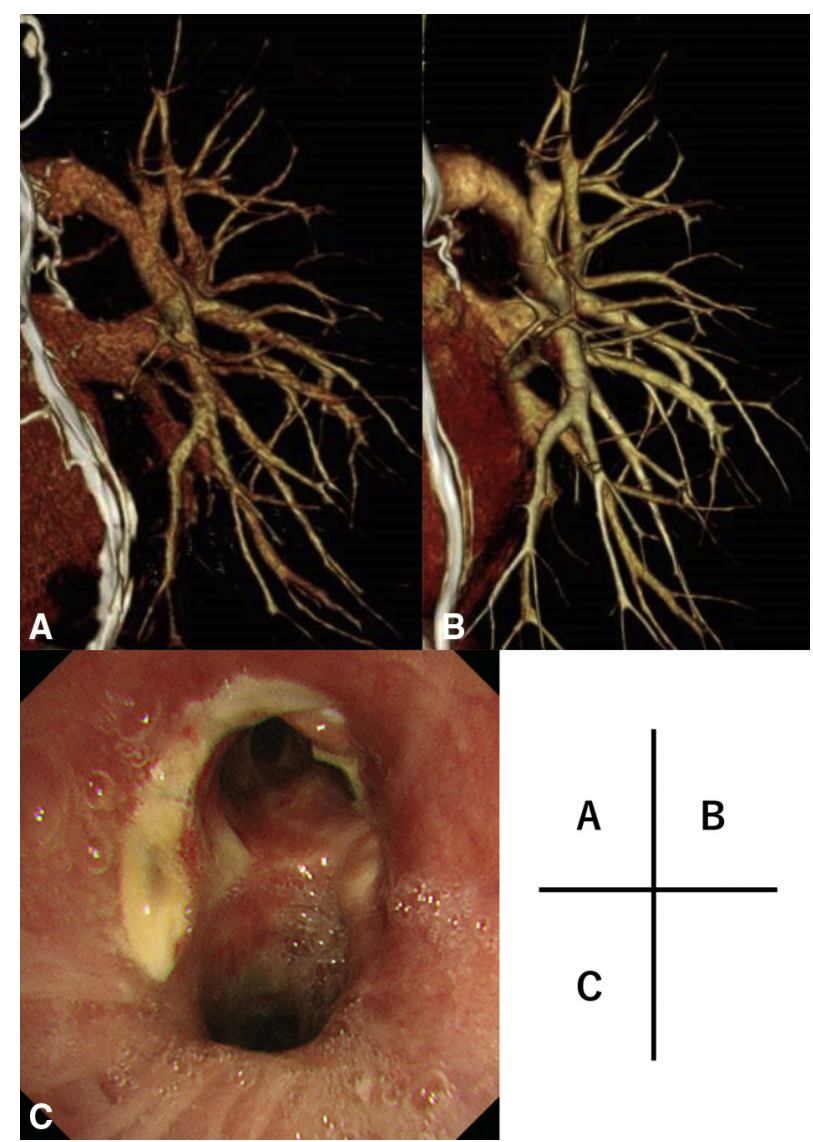

FIGURE 2. A, 3D-CT angiography image obtained at 1 week after the operation. The anastomosis site of the pulmonary artery was behind the bronchus. B, 3D-CT angiography performed 2 months later showed good patency and dilatation of the pulmonary artery and vein. C, Bronchoscopy image of anastomosis site of bronchus.

anastomosis of the left atrium was performed with 4-0 PROLENE sutures in the usual manner. A conduit between the left atriums was not used, because they were in close proximity to each other.

\section{Postoperative Course}

The postoperative course was uneventful. Threedimensional computed tomography was performed 1 week and again 2 months after surgery, with representative images shown in Figure 2 and Video 2. At 22 months after the procedure, the patient was alive without complications related to inverted lung transplantation.

\section{DISCUSSION}

Inverted lung transplantation provides a greater opportunity for single-lung transplantation in patients with uneven disease laterality. This is the first known report of right-toleft inverted single-lung transplantation, whereas Couetil and colleagues ${ }^{2}$ previously reported a left-to-right lung transplant. We have treated 3 patients, including the present

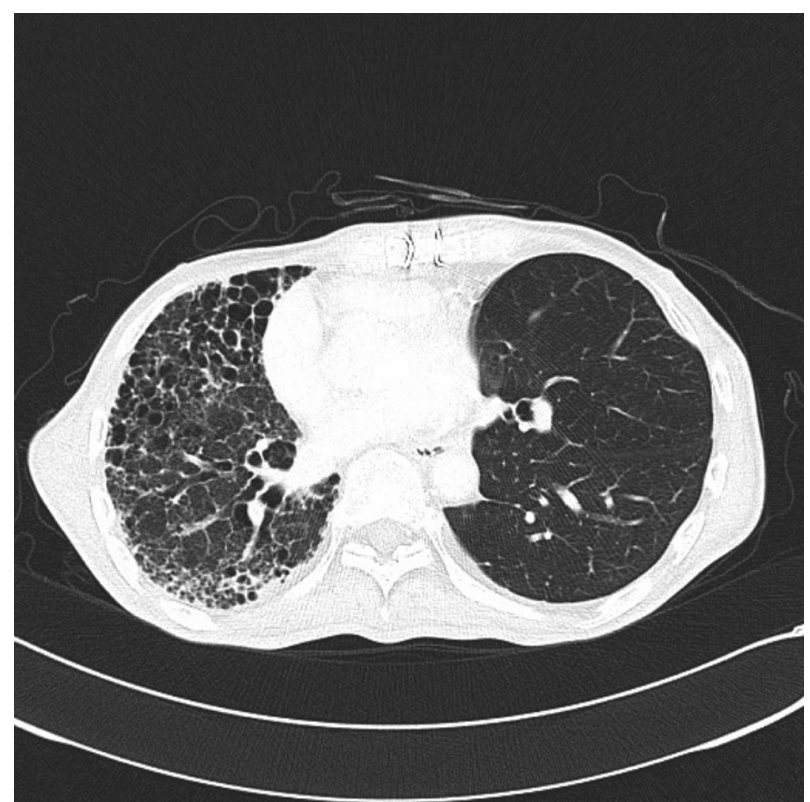

VIDEO 2. Postoperative chest computed tomography scan performed 2 months after lung transplantation. Video available at: https://www. jtcvs.org/article/S2666-2507(20)30557-5/fulltext.

patient, with inverted lung transplantation and obtained good results. It is considered that an inverted transplant procedure is a good therapeutic option for patients on the waiting list for single lung transplantation with uneven disease laterality.

For inverted lung transplantation, because the axis angles of the bronchi differ, we prefer the use of a method for oblique end-to-end bronchial anastomosis, ${ }^{1}$ as a donor bronchus-shortening technique decreases the risk of peripheral bronchial ischemia. ${ }^{3}$ Although the bronchial anastomosis was not covered in the present case, such additional covering may be beneficial to prevent ischemia-induced complications.

During the transplantation procedure, we routinely employ transesophageal echocardiogram imaging to assess flow in the anastomosed vessels. Monitoring both the flow with transesophageal echocardiogram and changes in pulmonary artery pressure is useful for evaluation of atypical vessel anastomosis.

\section{References}

1. Weder W, Inci I, Korom S, Kestenholz PB, Hillinger S, Eich C, et al. Airway complications after lung transplantation: risk factors, prevention and outcome. Eur J Cardiothorac Surg. 2009;35:293-8.

2. Couetil J-P, Argyriadis PG, Tolan MJ, Achkar A, Carpentier AF. Contralateral lung transplantation: a left lung implanted in the right thorax. Ann Thorac Surg. 2001;72:933-5.

3. van Berkel V, Guthrie TJ, Puri V, Krupnick AS, Kreisel D, Patterson A, et al Impact of anastomotic techniques on airway complications after lung transplant. Ann Thorac Surg. 2011;92:316-21. 